\title{
Modeling and Simulation of a High-speed Maglev Vehicle on an Infinite Elastic Guideway
}

\author{
Georg Schneider $^{1}$, Patrick Schmid ${ }^{1}$, Florian Dignath ${ }^{2}$, Peter Eberhard ${ }^{1}$ \\ ${ }^{1}$ Institute of Engineering and Computational Mechanics \\ University of Stuttgart \\ Pfaffenwaldring 9, 70569 Stuttgart, Germany \\ [georg.schneider, patrick.schmid, peter.eberhard]@itm.uni-stuttgart.de \\ 2 thyssenkrupp Transrapid $\mathrm{GmbH}$ \\ Moosacher Str. 58, 80809 Munich, Germany \\ florian.dignath@thyssenkrupp.com
}

\begin{abstract}
For magnetic levitation transport systems with pillared tracks, detailed simulation models of the coupled system of vehicle and guideway offer a valuable contribution to find a tradeoff between stiff/heavy guideway elements, required to keep disturbances small for the controller, and low material consumption. This work provides a novel model of a detailed rigid multibody Transrapid maglev vehicle with three sections moving along an infinite periodically pillared elastic guideway mapping the two-dimensional heave-pitch motion of the vehicle and the elastic bending of the guideway elements. The infinite guideway is realized by moving system boundaries, i.e., the same few Euler-Bernoulli beams representing the current track segment are used repeatedly to form an infinite sequence of guideway elements. The equations of motion of the elastic beams and the rigid multibody vehicle are obtained from the multibody modeling and simulation toolbox Neweul- $\mathrm{M}^{2}$. A detailed magnet model in combination with a model predictive control scheme are used for the first time in a large maglev vehicle model in this contribution. All model components are combined and coupled in a Simulink model. Simulation results show more severe overshoots and oscillations of the guideway deflection below the vehicle the faster the vehicle passes, which is resulting in bigger control errors and magnet motions at the rear end of the vehicle compared to the vehicle mid and front. Therefore, in contrast to previous presumptions, the most critical situations are to be expected at the rear end magnet, not at the front end.
\end{abstract}

Keywords: High-speed Maglev, Transrapid, Flexible Multibody System, Infinite Elastic Guideway, Moving System Boundaries.

\section{INTRODUCTION}

To this day, the only commercial high-speed magnetic levitation (maglev) train is implemented at the Shanghai Maglev Transportation line between Pudong International Airport and Longyang Road Station. It is based on the electromagnetic suspension (EMS) technology and runs with a maximum speed of $430 \mathrm{~km} / \mathrm{h}$ during rush hour. Currently, a new high-speed maglev train with a maximum speed of more than $600 \mathrm{~km} / \mathrm{h}$ is under development at the Chinese rolling stock manufacturer CRRC Qingdao Sifang Co., Ltd. [1]. A prototype of the future vehicle has already been presented to the public. The new high-speed maglev train aims to close the gap between current high-speed railway technology with top speeds of 300 to $350 \mathrm{~km} / \mathrm{h}$ and aircrafts traveling with speeds around $900 \mathrm{~km} / \mathrm{h}$. Therefore, the new maglev train offers a notable alternative for short to medium-haul flights regarding economic and ecologic aspects. For safety and ride comfort aspects, a perfectly rigid guideway would be desirable. However, with increasing stiffness requirements, 
production costs and material consumption for the guideway girders are also increasing. For finding a tradeoff, simulations and analyses with suitable models taking into account the dynamical behavior of the coupled system of guideway, vehicle, magnet, and controller provide valuable insights.

The dynamic interaction of maglev vehicles and their pillared track is investigated in numerous previous publications focusing on different aspects. Some publications focus on the bending of an elastic beam representing the guideway but simplify the vehicle by a point mass or single constant force $[2,3,4]$. Others exploit a detailed vehicle model, but the guideway is modeled by rigid elements $[5,6]$. In $[7,8,9]$, these approaches are combined by a rigid multibody vehicle crossing either a short series of elastic guideway elements or a single one, respectively. The simulation results from [9] show that for high speeds the elastic deformation of the guideway element causes a disturbance that influences the vehicle dynamics for several seconds after the vehicle has left the elastic guideway element. Thus, to predict the coupled system dynamics during a ride on a periodically pillared track, a model is required that allows the vehicle to travel a longer distance, including passing multiple elastic guideway elements. The idea of a guideway of infinite length represented by a finite number of guideway elements is already discussed in [10] but not elaborated in detail. Furthermore, as pointed out in [11], a more detailed vehicle model as, for example, described in $[5,6]$ is desirable to obtain accurate results.

The novel aspect of this contribution is the infinite elastic guideway formed by a repeating sequence of a few guideway elements combined with a detailed model of a complete Transrapid maglev vehicle consisting of three sections. Furthermore, this is the first complete vehicle model making use of the detailed magnet model from [12] and a model predictive control (MPC) scheme from [13], proving the usability of such kind of magnet models in combination with an MPC approach for the simulation of large maglev vehicle models. This novel model allows to investigate the differences in dynamics at the magnets at the front and rear end of the vehicle compared to the magnets in the middle. To the best of the authors' knowledge, such a detailed coupled system has not been modeled, investigated, and published before.

The subsequent sections are structured as follows. Section 2 describes the simulation model implemented and used in this contribution. It comprises the mechanical models of vehicle and guideway, magnet models and controllers, as well as the Simulink model of the coupled system. Interesting simulation results obtained from this model regarding the guideway dynamics, control accuracy, and the influence of guideway stiffness are presented and discussed in Sec. 3. Finally, a conclusion follows in Sec. 4.

\section{SIMULATION MODEL}

In this contribution, the multibody system approach is used to set up a two-dimensional model of a maglev vehicle moving along an infinite elastic guideway, mapping the heave-pitch motion and vertical guideway bending. Figure 1 visualizes the complete mechanical system in an undeformed state. In the following, the model components are explained in detail, especially the mechanical models of vehicle and guideway as well as the coupled system.

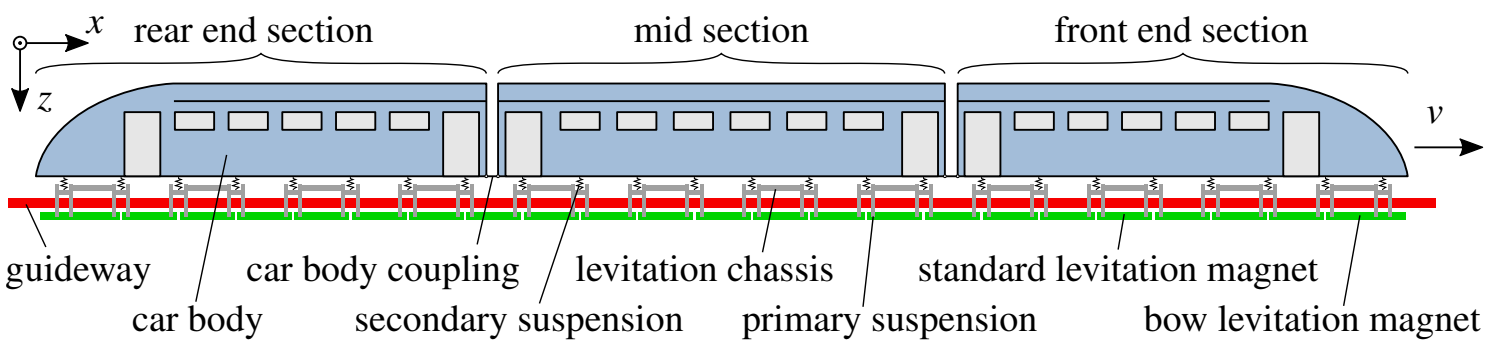

Figure 1. Components of the complete vehicle model on the guideway. 


\subsection{Mechanical Guideway Model}

Based on the model from [9], where the guideway is modeled as single elastic Euler-Bernoulli beam preceded and followed by a rigid track, in this contribution the track model is extended to represent a regularly pillared elastic guideway of infinite length. For efficiency reasons, the number of system states is kept small by using just a few guideway elements and applying the concept of moving system boundaries as described in [10]. The basic idea of this concept is to consider only as many guideway elements as coupled by the vehicle and use them repeatedly. To obtain the few required guideway elements, a single guideway element is copied multiple times, thus all guideway elements are identical.

\subsubsection{Single Guideway Element}

The basic unit for the guideway model, a single guideway element, is modeled as simply supported single-span Euler-Bernoulli beam with a length of $24.768 \mathrm{~m}$ discretized by 24 finite beam elements and reduced to its first three eigenmodes. For a description of the beam representing a single guideway element please refer to [9], where detailed explanations are provided about how moving magnet forces are applied to the beam by means of equivalent nodal forces and torques at its nodes, and how nodal coordinates are interpolated to get the deflections at arbitrary positions between the nodes using Hermite interpolation polynomials.

\subsubsection{Moving System Boundaries}

The complete infinite track is represented by a small number of identical guideway elements described above, which are used repeatedly. The minimum number of required guideway elements depends on the number of elements coupled by the moving vehicle. In general, the maximum number of guideway elements covered - at least partially - by the vehicle is

$$
n_{\text {covered }}=\left\lceil l_{\text {vehicle }} / l_{\text {beam }}\right\rceil+1
$$

with the overall vehicle length $l_{\text {vehicle }}$, single beam length $l_{\text {beam }}$, and the ceiling function $\lceil x\rceil$, mapping $x$ to the least integer greater than or equal to $x$. Taking into account only the guideway elements which are coupled by the vehicle is sufficient, because the guideway elements are decoupled from each other. Therefore, there is no guideway dynamics ahead of the foremost covered beam, and the vibrating beams behind the rearmost covered one do not have any influence on the vehicle. Nevertheless, the implementation of the coupled model in Simulink requires two more guideway elements. First, to allow for simulations with non-constant velocity, the times when guideway elements must be shifted to be reused is determined based on the vehicle position and not by the simulation time. That is, as soon as the vehicle front leaves a beam there must be the next one already available in front of it. Due to the block execution order in Simulink and also due to graphical representation reasons, the next beam shall be in its position already before the vehicle enters it. Therefore, an additional guideway element has to be available in front of the foremost covered one to avoid the vehicle "falling off" the track. Second, the states of a guideway element must be reset to their nominal values to reuse it after it has been passed by the vehicle. Resetting the system states in Simulink requires the subsystem of the respective guideway element to be disabled for at least one time step, which reduces the number of available guideway elements during that time. For implementation efficiency reasons, it is advantageous to keep the number of enabled guideway elements constant at all times. Therefore, the passed guideway element remains disabled not just for one time step, but for the complete time it takes for the vehicle front to cross a beam, and an additional guideway element is added to compensate for the disabled one. Thus, the number of required guideway elements for the simulation model is

$$
n_{\text {required }}=n_{\text {covered }}+2 \text {. }
$$

In Fig. 2, the concept is visualized with an example. Here, the maximum number of guideway elements covered by the vehicle is $n_{\text {covered }}=7$ as shown in the first configuration at time $t_{1}$. An 


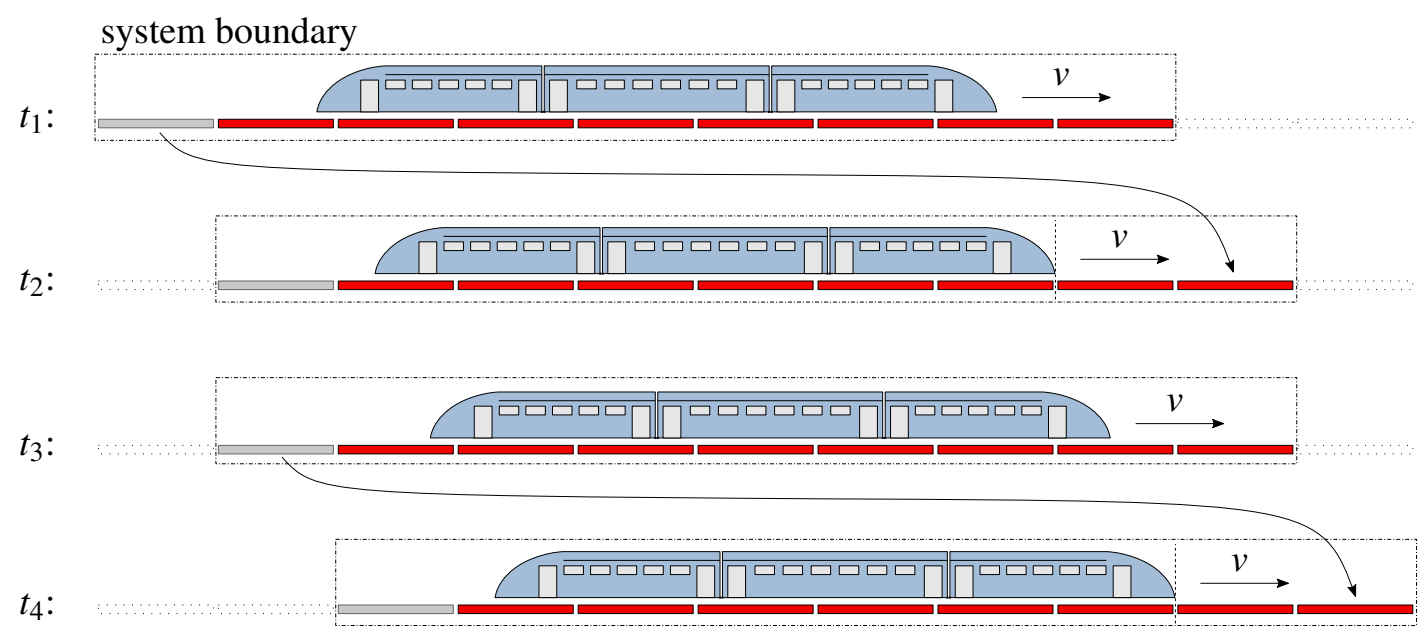

Figure 2. Concept of moving system boundaries: A small number of guideway elements is used repeatedly to realize an infinitely long elastic guideway and keep the number of system states small at the same time.

eighth guideway element is already in position in front of the vehicle, still uncovered but ready to be entered. These eight guideway elements colored red are enabled in the Simulink model. A ninth guideway element, colored gray, is located behind the rearmost enabled one and is disabled in Simulink. These nine guideway elements together with the vehicle define the system boundary. As soon as the vehicle enters the so far foremost beam, the disabled guideway element from the rear is re-enabled, its states are reset, and it is shifted from behind the vehicle to the very front, as shown in the second configuration at time $t_{2}$. The new rearmost, now uncovered guideway element is getting disabled. As long as the vehicle front does not reach the next beam, the guideway element positions and thus the system boundaries remain unchanged, see the configuration at time $t_{3}$. When the vehicle enters the next beam at time $t_{4}$, the shifting process is repeated. So the model boundaries, given by the rear and front end of the currently considered track segment, are shifted along the track together with the moving vehicle and an infinitely long elastic guideway is realized while the number of system states is kept small. If guideway vibrations should be analyzed in detail, an arbitrary number of additional guideway elements may be added behind the vehicle to observe the decay in guideway oscillations after a vehicle has passed. For the analysis of the vehicle dynamics, additional guideway elements behind the vehicle are not necessary.

\subsection{Mechanical Vehicle Model}

The vehicle model is based on the descriptions in $[5,6]$, representing a detailed two-dimensional rigid multibody model of the maglev vehicle Transrapid mapping the heave-pitch motion in the $x$ $z$-plane. Left and right side ( $y$-direction) of the system are summed up. Figure 1 gives an overview of the mechanical vehicle components.

\subsubsection{Kinematics}

The model consists of three sections: a rear end section, a mid section, and a front end section. Each section consists of rigid bodies for a car body, four levitation chassis, and seven or eight levitation magnets, respectively. Each section has the length of eight standard levitation magnets, but the magnets are arranged in such a way that the neighboring levitation chassis of two sections are connected by a magnet. At the front and rear end of the vehicle, bow levitation magnets are installed that are longer and have more poles. Thus, they have a shifted center of gravity (COG) and higher mass and inertia compared to a standard levitation magnet. The levitation chassis at the 
rear and front end of the vehicle also differ from the others, since they have a cladding mounted resulting in a shifted COG as well as higher mass and inertia. In addition, the rear and front end section car bodies have shifted COGs and less mass and inertia compared to the mid section due to different geometries.

The car bodies' kinematics are defined with respect to an inertial system. The kinematics of the levitation chassis and levitation magnets are defined with respect to their respective car body. The levitation magnets connecting two sections belong to the respective rearward section, i.e., the levitation magnet between rear end section and mid section is defined with respect to the rear end car body, and the levitation magnet between mid section and front end section is defined with respect to the mid car body.

Each of the rigid bodies has two degrees of freedom (DOF): a translational one in $z$-direction and a rotational one about the $y$-axis. For three car bodies, twelve levitation chassis, and 23 levitation magnets this results in $(3+12+23) \cdot 2=76$ DOF for the complete vehicle.

\subsubsection{Force Elements}

The bodies are coupled by force elements. Elastomer element couplings, represented by linear spring-damper combinations in the model, connect the car bodies. The car bodies are supported on the levitation chassis by the soft secondary suspension, which is realized by air spring rockers at the real vehicle, see [6], for example. Their purpose is the decoupling of the car body dynamics from the higher frequency vibrations of magnets and chassis. In the model, the mass and inertia of the rockers is neglected, but their leverage is taken into account for the computation of the stiffness of the air springs that are modeled as linear spring-damper elements. The levitation chassis are supported on the magnets by very stiff elastomer elements, also modeled as linear spring-damper combinations. Finally, the magnets are coupled to the guideway by the magnet forces that attract the magnets to the guideway from below. The magnet forces are computed by the magnet model described in the subsequent section and they are implemented as inputs to the mechanical model. As the used magnet model computes one concentrated force per half magnet, two force elements per standard levitation magnet are implemented. The bow levitation magnets are simplified by three half magnets and thus three magnet forces are applied there.

\subsection{Magnet Model}

The electromagnet dynamics of the levitation magnets is considered by means of the magnet model presented in [12], including the effects of magnetic reluctances, fringing and leakage flux, magnetic saturation, and eddy currents. Two models are available: a detailed one of a complete magnet computing forces at each of the twelve magnet poles, and a simplified one of a half magnet summing up the forces in a single concentrated substitute force per half magnet. The detailed model is described by a set of differential-algebraic equations (DAEs) formulated in terms of the magnetic flux. Solving a set of DAEs is computationally intensive and, therefore, unsuitable for simulating large vehicle models with numerous magnets involved. The simplified model, however, is described by a single ordinary differential equation (ODE), or two ODEs for a complete magnet, respectively. The simplified model is derived from the detailed model in a numerical model reduction procedure yielding characteristic diagrams for the inductance, its derivative, and the force that are exploited by the simplified model. Both model variants have nearly the same input-output structure and almost the identical static and dynamic behavior, but the simplified one allows a tremendous speedup of two orders of magnitude compared to the detailed one. This makes it usable for the model in this contribution with 21 standard levitation magnets and two bow levitation magnets, equivalent to 48 half magnets in total. The magnet model is validated for a Transrapid's levitation magnet, i.e., the simulation results obtained with it in [12] match measurements very well nearly across the whole operation range of the magnet. 


\subsection{Controller}

Maglev systems based on the EMS system, i.e., working with attractive magnet forces, are inherently unstable due to the reciprocal relation of magnet force and air gap. The electromagnets must be actively controlled to keep the air gap in a safe range to avoid physical contact between the vehicle and the guideway. Each half magnet has its own gap measurement unit (GMU) and is controlled individually by its own magnet control unit, which provides the voltage for the magnet based on the gap, acceleration, and current measurements.

A promising approach to control high-speed maglev vehicles is nonlinear MPC as stated in [14], because system constraints and the nonlinear nature of the magnet control system can be considered actively in the control design. However, different quasi-static loads for the individual magnet parts or just plant-model mismatch require the use of an offset-free MPC scheme. In this contribution, the integral error approach from [13] is used, which is found suitable for application for a Transrapid vehicle. See [13] for a detailed discussion and comparison to other offset-free MPC approaches for the magnet control system. By varying the cost functional's weighting matrices of the underlying optimal control problem being solved at each time step to determine the control input, the speed of the closed-loop system can be adapted in an intuitive manner. In this paper, one parameterization of the weighting matrices and the desired gap is used for all vehicle velocities.

\subsection{Coupled System}

The mechanical models of the vehicle and the guideway, respectively, are set up with the Matlabbased multibody modeling and simulation toolbox Neweul- $\mathrm{M}^{2}$ [15]. With Neweul- $\mathrm{M}^{2}$, the equations of motion are computed in symbolic form and written to Matlab files for numerical evaluation. Furthermore, the equations of motion are exported to $\mathrm{C}$ code and compiled as mex files, allowing to include them as S-functions in Simulink. The magnet and controller models are created with Matlab/Simulink as well. Therefore, all system components, i.e., the mechanics, the magnets, and the controllers, are coupled in Simulink. The Simulink model topology is shown in Fig. 3 and the variables used there are described in Tab. 1.

The equations of motion for the vehicle and guideway mechanics (yellow blocks) are included by means of the $\mathrm{S}$-functions that are exported automatically by Neweul- $\mathrm{M}^{2}$. From the outputs of the mechanical models, various air gaps and air gap velocities are computed by interpolating the nodal coordinates and velocities of the guideway $\boldsymbol{w}, \boldsymbol{\varphi}, \dot{\boldsymbol{w}}$, and $\dot{\boldsymbol{\varphi}}$ using Hermite polynomials as described in [9] and subtracting the results from the corresponding $z$-positions and velocities of the magnet at the concentrated substitute magnet forces and GMUs $z_{\mathrm{MF}}, \dot{z}_{\mathrm{MF}}$, and $z_{\mathrm{GMU}}$. The vector of air gaps at the GMUs $\boldsymbol{s}_{\mathrm{GMU}}$, vector of accelerations at the GMUs $\ddot{z}_{\mathrm{GMU}}$, and vector of currents $\boldsymbol{I}$, provided by the magnet models (summed up in the green block), are the inputs for the individual controllers (summed up in the blue block). The controllers, in return, provide the vector of magnet voltages $\boldsymbol{U}$ serving as input for the magnet models together with the vectors of air gaps and air gap velocities at the concentrated substitute magnet forces $\boldsymbol{s}_{\mathrm{MF}}$ and $\dot{\boldsymbol{s}}_{\mathrm{MF}}$. In addition to the vector of magnet currents $\boldsymbol{I}$, the magnets primarily provide the vector of concentrated substitute magnet forces $\boldsymbol{F}_{\mathrm{MF}}$ coupling the vehicle and guideway subsystems. This signal is used directly as input to the vehicle mechanics. For application of the magnet forces to the guideway, $\boldsymbol{F}_{\mathrm{MF}}$ has to be replaced by a vector of nodal forces and torques $\boldsymbol{F}_{\text {nodal }}$. This is realized by a decomposition with the aid of Hermite polynomials distributing the concentrated substitute magnet forces to equivalent nodal forces and torques acting at neighboring beam nodes as described in [9]. The $x$-position of the front end car body over time $\boldsymbol{x}_{\mathrm{CB}}$ is provided by a workspace variable and serves as input to the vehicle mechanics block. Finally, the $x$-positions of the concentrated substitute magnet forces $\boldsymbol{x}_{\mathrm{MF}}$ are passed to the guideway block. From these positions, quantities like the position of the vehicle front or the overall length of already passed guideway elements are computed, which are required for the correct arrangement of the few available guideway elements to form the track segment within the current system boundaries. 


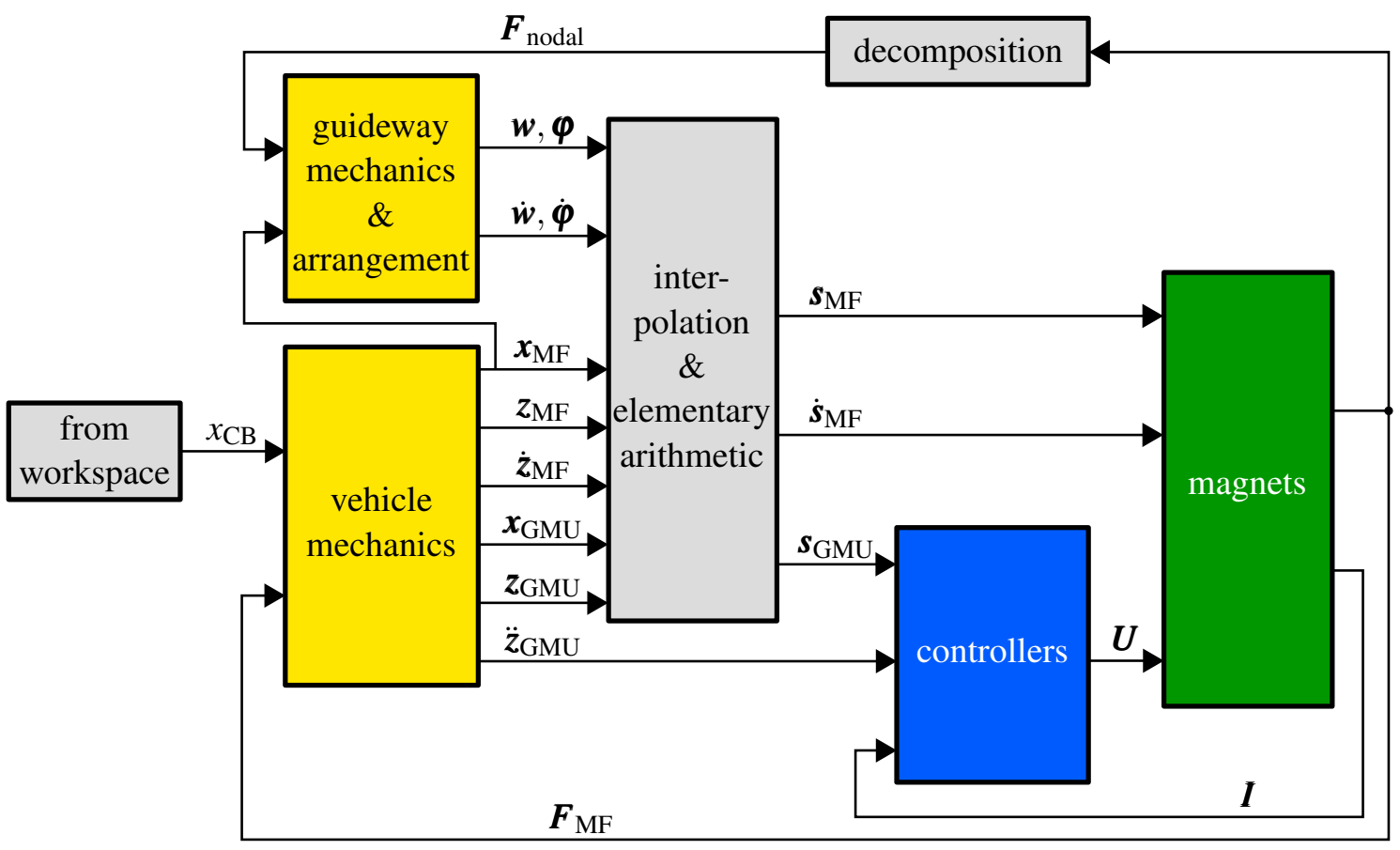

Figure 3. Schematic setup of the coupled model in Simulink. Descriptions of all variables used are given in Tab. 1.

Table 1. List of variables used in Fig. 3.

\begin{tabular}{ll}
\hline variable & description \\
\hline$x_{\mathrm{CB}}$ & x-position of front end car body \\
$\boldsymbol{x}_{\mathrm{MF}}$ & vector of $x$-positions of concentrated substitute magnet forces \\
$z_{\mathrm{MF}}$ & vector of $z$-positions of magnets at concentrated substitute magnet forces \\
$\dot{z}_{\mathrm{MF}}$ & vector of $z$-velocities of magnets at concentrated substitute magnet forces \\
$\boldsymbol{x}_{\mathrm{GMU}}$ & vector of $x$-positions of gap measurement units \\
$z_{\mathrm{GMU}}$ & vector of $z$-positions of magnets at gap measurement units \\
$\ddot{z}_{\mathrm{GMU}}$ & vector of $z$-accelerations of magnets at gap measurement units \\
$\boldsymbol{w}, \boldsymbol{\varphi}$ & vectors of nodal coordinates (translational and rotational) of beams \\
$\dot{\boldsymbol{w}}, \boldsymbol{\varphi}$ & vectors of nodal velocities (translational and rotational) of beams \\
$\boldsymbol{s}_{\mathrm{MF}}$ & vector of air gaps at concentrated substitute magnet forces \\
$\dot{\boldsymbol{s}}_{\mathrm{MF}}$ & vector of air gap velocities at concentrated substitute magnet forces \\
$\boldsymbol{s}_{\mathrm{GMU}}$ & vector of air gaps at gap measurement units \\
$\boldsymbol{U}$ & vector of magnet voltages \\
$\boldsymbol{I}$ & vector of magnet currents \\
$\boldsymbol{F}_{\mathrm{MF}}$ & vector of concentrated substitute magnet forces \\
$\boldsymbol{F}_{\mathrm{nodal}}$ & vector of nodal forces and torques \\
\hline
\end{tabular}




\section{SIMULATION RESULTS}

For the simulations in this contribution, the mechanical vehicle model and the magnet model described in Sec. 2 are parameterized according to the so-called TR08, the Transrapid vehicle which is the predecessor of the vehicle running at the Shanghai Maglev Transportation line. The MPC scheme uses the such parameterized models for its internal controller model. The guideway beams are parameterized with data from the first generation of concrete guideway at the $30 \mathrm{~km}$ test facility in northern Germany (TVE) as provided by [11]. No pre-buckling of the beams is modeled, but they are undeflected in the unloaded state when their own weight is the only applied force acting on them.

In the following, the presented model is used to simulate a Transrapid vehicle with three sections moving along a periodically pillared infinite elastic guideway with various velocities. Different model characteristics are examined such as the guideway dynamics below a passing vehicle as well as the air gap's control accuracy and the magnet motion at different positions along the vehicle. Moreover, the influence of different guideway stiffness values on the mechanical magnet dynamics is analyzed.

\subsection{Guideway Dynamics}

Figure 4 shows the deflection of a single guideway element at mid span $w_{\text {mid }}$ versus the position of the foremost magnet force $x_{\mathrm{MF} \text {,front }}$, while the vehicle is passing with different velocities. At $x_{\mathrm{MF} \text {,front }}=0$, the foremost magnet force enters the beam and leaves it again at the first dashed vertical line. Thus, the complete beam is covered by the vehicle until the rearmost magnet force enters the beam at the second dashed vertical line. Finally, at the last dashed vertical line the rearmost magnet force leaves the beam, which is then uncovered and can oscillate freely.

In contrast to the corresponding but preliminary study in [9], the guideway element is now completely covered by subsequent vehicle sections for some time, which allows the analysis of its dynamic behavior under the load of the passing vehicle. In the quasi-static case, here represented by a vehicle speed of $18 \mathrm{~km} / \mathrm{h}$, the deflection smoothly increases with increasing overlap of vehicle and beam, reaching a value of nearly $6 \mathrm{~mm}$ when the beam is covered completely, and decreases again to zero when the vehicle leaves. The higher the velocity of the passing vehicle, the more severe overshoots and oscillations are visible, meaning more challenging disturbances for the controllers at the mid and rear vehicle sections to deal with.

The dynamics of the deflections in Fig. 4 can be explained as follows. The bending of an EulerBernoulli beam is described by a partial differential equation of second order in time, see [10], for example. For constant vehicle velocity, the excitation of the beam caused by the increasing load of the entering vehicle adopts the shape of a ramp signal. The same holds for the unloading

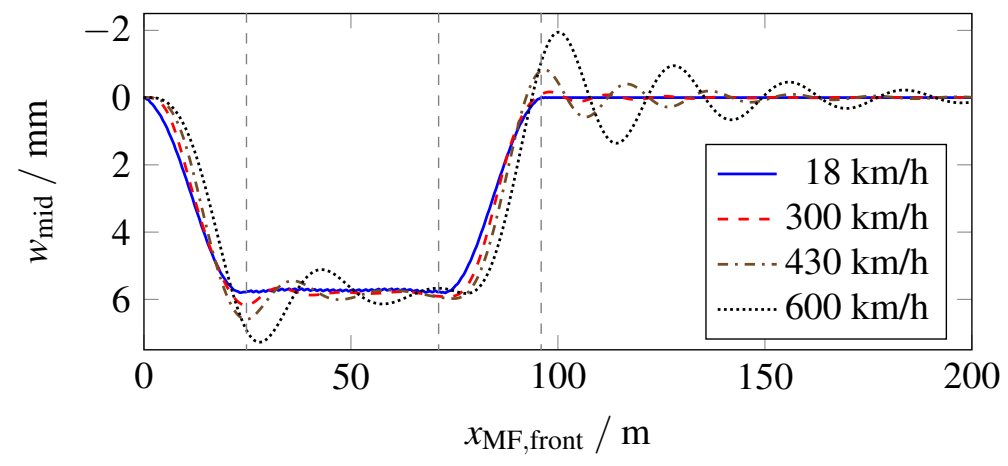

Figure 4. Deflection of a single guideway element at mid span versus position of the foremost magnet force for various velocities. 
process with a decreasing load when the vehicle leaves the beam. The ramp shape of the excitation approximates more and more a step shape the faster the vehicle is running. Thus, the overshoot and oscillations visible in the beam deflection for high velocities approximately represent a step response of a second order system. A higher velocity, i.e., a more step-like excitation, results in a stronger overshoot and oscillations of the beam with its eigenfrequency, which in turn induce vibrations to the vehicle.

Based on these results, for such high velocities a higher guideway stiffness is strongly recommended to reduce the guideway deflections and thus make the control task easier. However, this increases the production effort for the guideway, which highlights the necessity of finding a tradeoff between acceptable effort and girder elasticity manageable by the magnet controller.

\subsection{Air Gap Control Accuracy and Magnet Motion Along the Vehicle}

This section investigates the control accuracy and the magnet motion at different positions along the vehicle and for different vehicle velocities. From a control design perspective, there must be a compromise to keep the air gap within a safe range and simultaneously reduce the magnet motion to improve the ride comfort. The relative air gap control error $\Delta s / s_{\text {des }}=\left(s_{\text {meas }}-s_{\text {des }}\right) / s_{\text {des }}$, i.e., the relative deviation of the measured air gap $s_{\text {meas }}$ from the desired air gap $s_{\text {des }}$ measured at the GMUs of magnets located at the very rear end, in the middle, and at the very front end of the vehicle, respectively, are plotted in the upper row of Fig. 5. The second row of Fig. 5 shows the normalized absolute magnet motion $z / z_{\text {nom }}$ with the nominal magnet $z$-position $z_{\text {nom }}$ being at $s_{\text {des }}$ below the undeformed beam.

At all three considered positions along the vehicle, the air gap control error grows with increasing vehicle speed. This comes along with an increasing phase shift of the magnet motion with respect to the guideway deflection with increasing velocity. The faster the vehicle passes, the farther behind the point where two guideway elements adjoin is the point where the magnet reaches its highest position. One reason for these effects is more oscillating guideway dynamics, see Fig. 4.
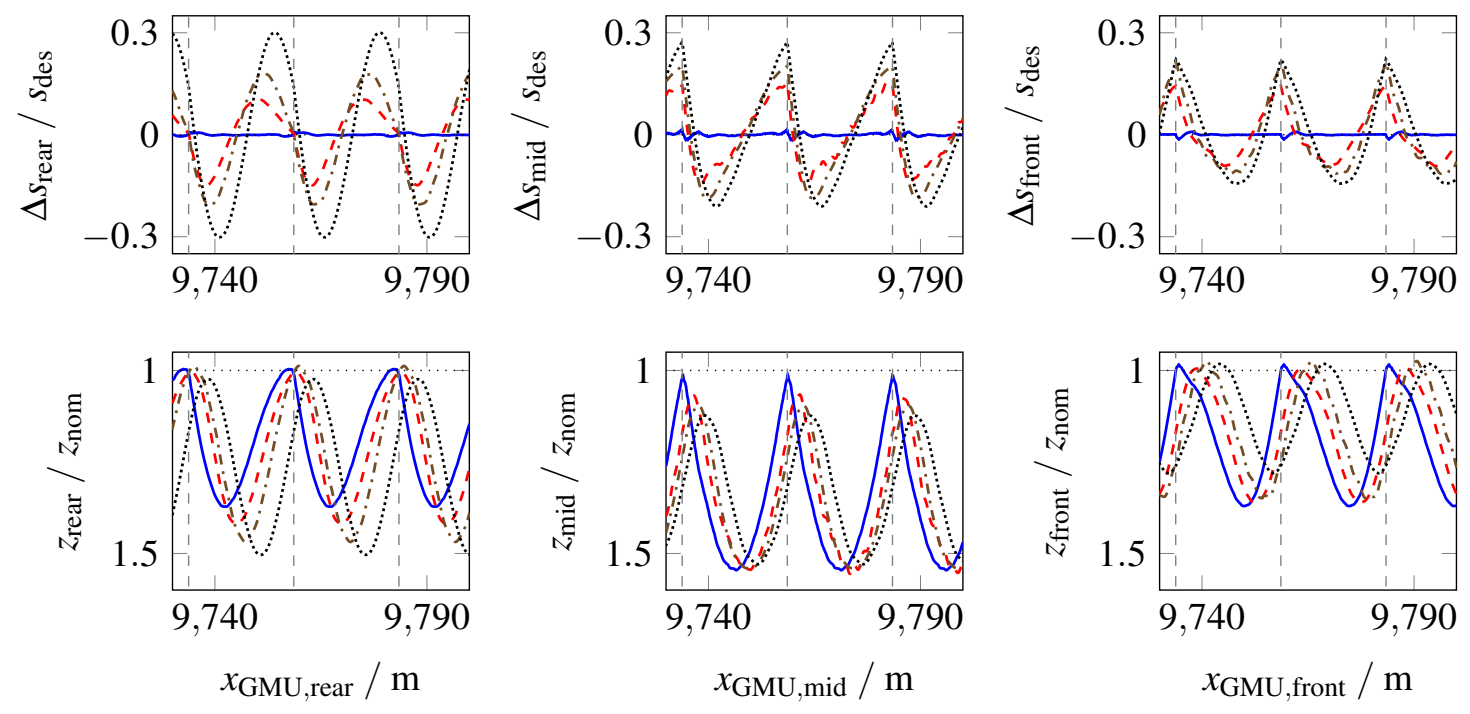

$18 \mathrm{~km} / \mathrm{h}---300 \mathrm{~km} / \mathrm{h}-\cdots 430 \mathrm{~km} / \mathrm{h} \cdots \cdots \cdots \cdot 600 \mathrm{~km} / \mathrm{h}$

Figure 5. Relative air gap control error and normalized absolute magnet $z$-positions at GMUs of magnets at the very rear end, in the middle, and at the very front end of the vehicle, respectively. Vertical dashed lines mark the positions where one guideway element ends and the next one begins. 
Moreover, the controller has to deal with a higher frequency of disturbances, but magnetic inductance and inertia forces regarding the mechanics are limiting factors for how fast the magnet suspension system can respond to changing air gaps.

Especially for high velocities, the biggest air gap control errors occur at the rear end of the vehicle, while the best control accuracy is achieved at the vehicle front. The magnet's $z$-motion is relatively small at the front compared to the mid and rear end positions as well. An explanation for this observation is that the vehicle front always enters a resting, undeflected guideway element, while magnets in the middle and rear part enter an already deformed and possibly oscillating beam. Therefore, the kink in the bending line at the positions where two guideway elements adjoin is less severe for the magnets in the front area of the vehicle than in the middle part. Furthermore, the guideway deflections for the controller to deal with at the vehicle front are smaller than at the middle and rear end magnets, since the girder is initially unloaded and thus the bending is still increasing while the vehicle front passes. This is also the reason for the effect revealed by the plots in the second row of Fig. 5, that the magnet motion depends on the vehicle speed, but differently for rear end, middle, and front end positions. While the amplitude of the oscillations increases with velocity at the rear end, it decreases slightly in the middle and at the front end. However, the smaller amplitudes for the vertical magnet motion at the middle and front end at higher velocities come along with higher air gap amplitudes because of the limited performance of the magnet suspension system. The vehicle rear end, where the biggest deviations from the desired air gap occur, seems to be the most critical part regarding a contact between vehicle and guideway. However, in the simulations for this contribution the air gaps are in a non-critical range even for high velocities highlighting the performance of the MPC approach used for the magnet controllers.

\subsection{Influence of Guideway Stiffness}

In this section, the influence of the guideway stiffness on the air gap control error and vertical magnet motion is analyzed by varying the elasticity of the modeled Euler-Bernoulli beam. Young's modulus $E$ takes the values $75 \%, 100 \%, 125 \%$, and $150 \%$ of the value $E_{0}$ representing the first generation of concrete guideway at the TVE used for the studies investigated above. The upper row of Fig. 6 shows the maximum relative air gap control error $\max (|\Delta s|) / s_{\text {des }}$ from Fig. 5 for the different stiffness values plotted over the vehicle speed at the rear end, middle, and front end of the vehicle for different velocities. In analogy, the amplitude of magnet motion $\Delta z=z_{\max }-z_{\min }$ normalized with $z_{\text {nom }}$ is plotted in the second row of Fig. 6. The values $z_{\max }$ and $z_{\min }$ are the maximum and minimum, respectively, of the magnet $z$-position as shown in the second row of Fig. 5. For $v=600 \mathrm{~km} / \mathrm{h}$ and $E / E_{0}=75 \%$, the simulation becomes unstable quickly at the beginning, thus no values are contained in the plots for this parameter combination.

In general, both the maximum air gap control error and the amplitude of magnet motion decrease with increasing guideway stiffness, because the maximal guideway deflection decreases as well. Looking at different velocities, at the rear end the effect of elasticity variation on the magnet motion is stronger for higher velocities, whereas at the middle and especially at the front end it is nearly equal for all analyzed velocities except for $600 \mathrm{~km} / \mathrm{h}$, for which it is even smaller. This is caused by the more severe guideway oscillations that the rear end magnet has to deal with while it leaves the beam. The quite sudden unbending of the beam for high vehicle speeds leads to an overshoot, see Fig. 4, meaning that the beam is bent upwards while the rear end magnet is still under it. The small influence of elasticity variation at the front end for $600 \mathrm{~km} / \mathrm{h}$ is also clearly visible in the maximum air gap control error. 

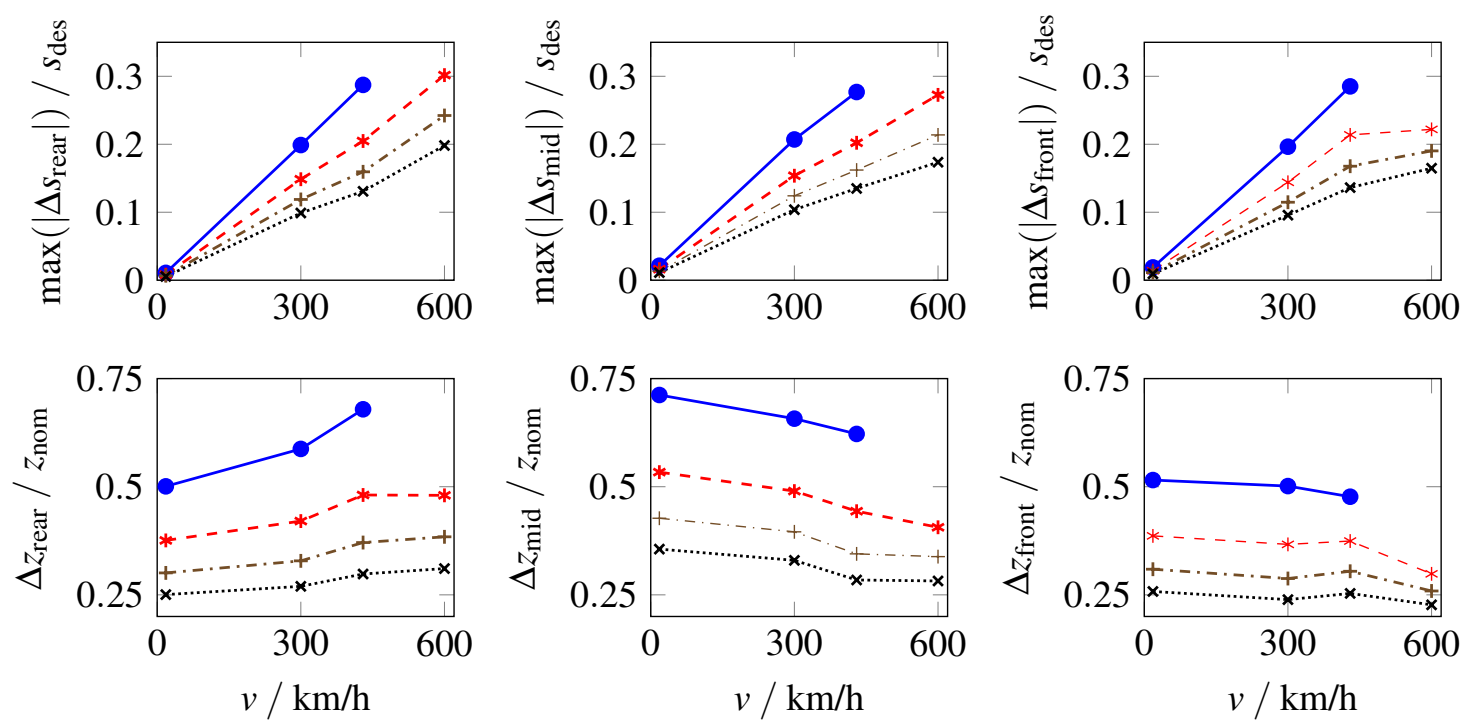

$75 \%-*-100 \%-+\cdot \cdot 125 \% \cdots * \cdots \cdot 150 \%$

Figure 6. Maximum air gap control error and amplitude of vertical magnet motion at the very rear end, in the middle, and at the very front end of the vehicle, respectively, for different guideway elasticity values $E / E_{0}$ plotted over different velocities.

\section{CONCLUSIONS}

The requirements for the guideway of a high-speed maglev system are contradictory. For the vehicle's magnet suspension system, girders would be preferable that are as stiff as possible to keep the disturbances due to bending small. On the other hand, this goes along with intensive material consumption and expensive production costs, but economic viability is essential for competitiveness with other transportation systems. This publication makes a contribution towards finding a tradeoff between these opposing requirements using a detailed model of a Transrapid vehicle with three sections traveling on an infinite elastic guideway mapping the two-dimensional heave-pitch motion of the vehicle. The system dimension is kept small due to system boundaries moving along with the vehicle allowing to reuse the same few guideway elements for the guideway again and again. The faster the vehicle moves, the more severe are overshoot and oscillations of the guideway deflection below the passing vehicle resulting in bigger magnet motions and air gap control errors at the middle and rear end of the vehicle compared to the vehicle front end. A remarkable new result is that controlling the vehicle rear magnets seems to be the most critical part at high speeds due to relatively intense guideway dynamics at the rear end. The situation at the vehicle front is even less critical than at the middle of the vehicle, where the difference between the slopes of the two adjacent bending lines is largest because both beams are maximally deflected at this moment. The same tendency holds also for the influence of the guideway elasticity on the magnet motion. It is smaller at the vehicle front than at the middle and rear parts, but at all locations a higher stiffness is advantageous due to smaller guideway deflections.

For further investigations with this model, additional guideway disturbances like offsets at adjoining girders and at adjoining stator packs could be taken into account. However, the disturbance due to guideway deflection is dominating. Another scenario that can be investigated with this model is the failure of one or several levitation magnets, to investigate if neighboring magnets are able to take over the additional load. Furthermore, analyzing the relation between span-crossing frequency, beam eigenfrequency, and speed of the magnet control closed-loop system are planned to analyze potential resonance effects and favorable parameters regarding the ride comfort. 


\section{REFERENCES}

[1] Lin, G., Sheng, X.: Application and further development of maglev transportation in China. Transportation Systems and Technology 4(3) (2018) 36-43

[2] Popp, K., Schiehlen, W.: Dynamics of magnetically levitated vehicles on flexible guideways. In Pacejka, H.B., ed.: Proceedings of IUTAM Symposium on the Dynamics of Vehicles on Roads and Railway Tracks. Amsterdam, Swets and Zeitlinger (1976) 479-503

[3] Meisinger, R.: Simulation of maglev vehicles riding over single and double span guideways. Mathematics and Computers in Simulation 21(2) (1979) 197-206

[4] Meisinger, R.: Simulation of a single and double-span guideway under action of moving MAGLEV vehicles with constant force and constant gap. Sonderdruck Schriftenreihe der Georg-Simon-Ohm-Fachhochschule Nürnberg 14 (2002)

[5] Hägele, N., Dignath, F.: Vertical dynamics of the maglev vehicle transrapid. Multibody System Dynamics 21(3) (2009) 213-231

[6] Dellnitz, M., Dignath, F., Flaßkamp, K., Hessel-von Molo, M., Krüger, M., Timmermann, R., Zheng, Q.: Modelling and analysis of the nonlinear dynamics of the Transrapid and its guideway. In: Progress in Industrial Mathematics at ECMI 2010. Volume 17. Heidelberg, Springer (2012) 113-123

[7] Han, J.B., Han, H.S., Lee, J.M., Kim, S.S.: Dynamic modeling and simulation of ems maglev vehicle to evaluate the levitation stability and operational safety over an elastic segmented switch track. Journal of Mechanical Science and Technology 32(7) (2018) 2987-2998

[8] Wang, Z., Xu, Y., Li, G., Yang, Y., Chen, S., Zhang, X.: Modelling and validation of coupled high-speed maglev train-and-viaduct systems considering support flexibility. Vehicle System Dynamics 57(2) (2019) 161-191

[9] Schneider, G., Liang, X., Dignath, F., Eberhard, P.: Simulation of the maglev train transrapid traveling on a flexible guideway using the multibody systems approach. In Kecskeméthy, A., Geu Flores, F., eds.: Multibody Dynamics 2019. ECCOMAS 2019. Computational Methods in Applied Sciences. Volume 53. Cham, Springer (2020) 503-510

[10] Popp, K., Schiehlen, W.: Ground Vehicle Dynamics. Springer, Berlin (2010)

[11] Ren, S., Romeijn, A., Klap, K.: Dynamic simulation of the maglev vehicle/guideway system. Journal of Bridge Engineering 15(3) (2010) 269-278

[12] Schmid, P., Schneider, G., Dignath, F., Liang, X., Eberhard, P.: Static and dynamic modeling of the electromagnets of the maglev vehicle transrapid. IEEE Transactions on Magnetics 57(2) (2021) 1-15

[13] Schmid, P., Eberhard, P.: Offset-free nonlinear model predictive control by the example of maglev vehicles. In: Proceedings of 7th IFAC Conference on Nonlinear Model Predictive Control. Bratislava (July 2021)

[14] Schmid, P., Eberhard, P., Dignath, F.: Nonlinear model predictive control for a maglev vehicle regarding magnetic saturation and guideway irregularities. IFAC-PapersOnLine 52(15) (2019) 145-150

[15] Kurz, T., Eberhard, P., Henninger, C., Schiehlen, W.: From Neweul to Neweul-M²: Symbolical equations of motion for multibody system analysis and synthesis. Multibody System Dynamics 24(1) (2010) 25-41 